تصنيع و ررلسة الخواص البصرية لمرشح المثل الأخضر الجيلانيف

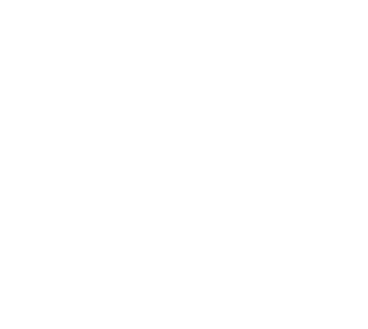

(تاريخ المستلام 15/ 12 / 2010 ؛ تاريخ القبول 14 / 3 / 2011)

\title{
ll
}

قم في هذا البهث تصنبع ودرلسة الخواص البصرية لمرشح رلنن جيلاتين بلستخدلم صـ ـبغة المثي لـ

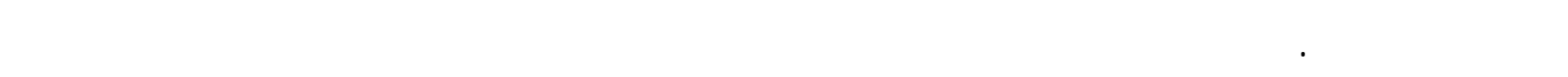

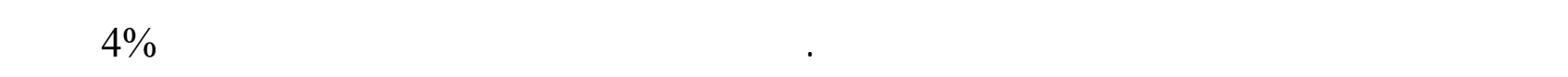

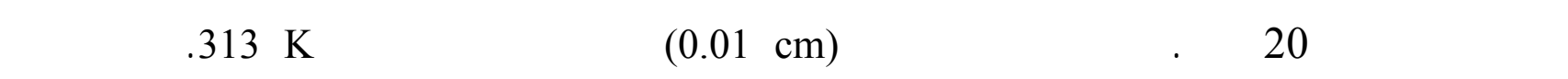

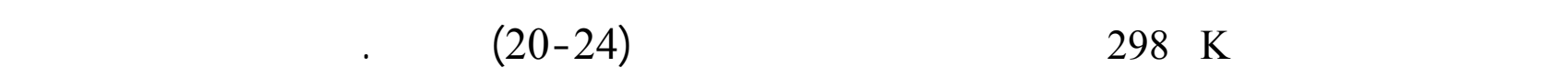

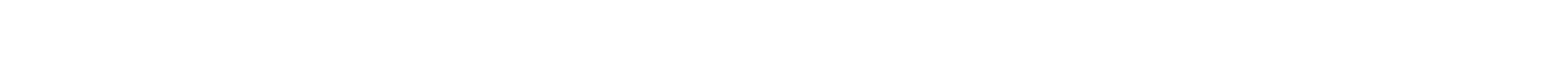

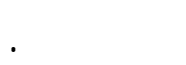
الهاملت الدالة : أصباغ، راتين جلاتين، مششحك .

\section{Fabrication and Study the Optical Properties for Methyl Green Gelatin Filter}

\author{
Soory A. Daher \\ Basic Science Branch \\ College of Agriculture \\ Mosul University
}

Ismail K. Abbas

Department of Physics

College of Science

Mosul University

\section{ABSTRACT}

In this work, an optical wratten gelatin filter was fabricated then its optical properties have been calculated, such as the filter thickness, aging, effect of protection glass plates, dye concentration and the period of mixing the gelatin with distill water at selective temperature. From this investigation, it was found that the best gelatin concentration is $4 \%$, also the preferable mixing time of gelatin with water is $20 \mathrm{~min}$. The preferable thickness is $0.01 \mathrm{~cm}$ at temperature $313 \mathrm{~K}$. The drying temperature is $298 \mathrm{~K}$ for period $20-24 \mathrm{hr}$. the optical characteristic of our filter is in good agreement with that standard filters. 
Keywords: Dyes, Wratten gelatin, Filters .

\section{الفمهة}

من المعروف أن مدى موجلت الطف الكهرومغنلطيسي ولسعة جدا بالاعتماد على التردد أو الط ـول

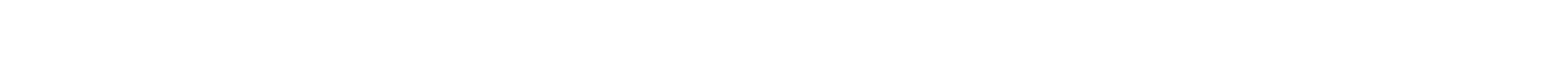

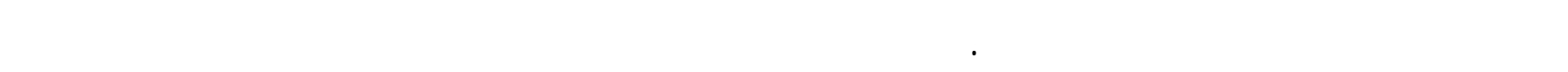
الموجلت المرئية وتسه الضوء ويحتوي على الألوان للسبعة حيث للعن القدرة على التعسس بها وتئ ـراوح

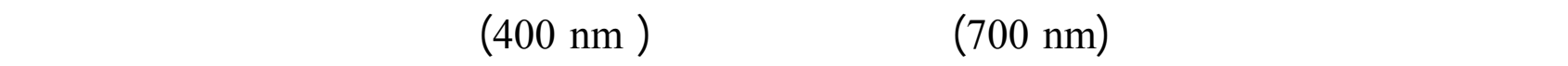

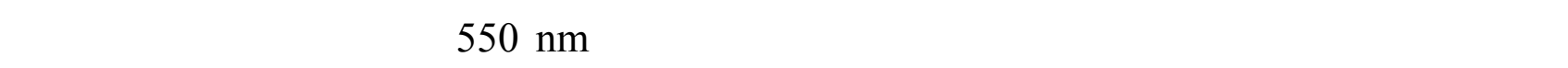
المخضر (Serway and Jewett, 2003). ليس من اللهوولة تعريف اللون، اللون كلمة تشير إلى لستجابة مجموعة عبن - ماغ لموجلت ال ـضوء

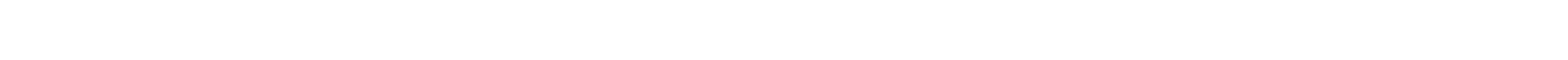

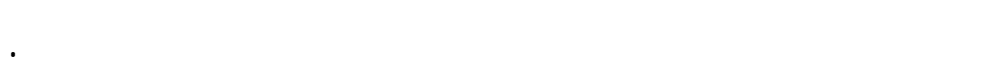
على الرغم من التعقيدات عن مفهوم اللون وإدرلكه ، يمكن تعينيه بصورة روفية دقيقة بثلاثة متغيرات فتط

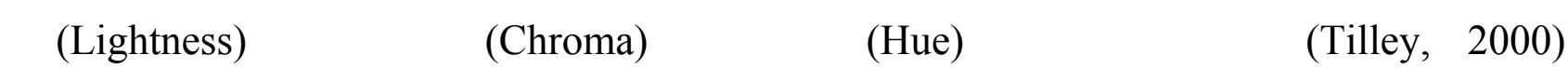
اللططوعية(Brightness). هنك أظمة تأنسق الألوان وتكون إما لحادية أو ثنائية أو ثلاثية الأبعاد وت ستخدم لتعيين الألوان بطريقة ظلمية وهنك العديد من الأظمة أهمها ظلم منسيل ، ظلم الألوان الطبيع مي، ظط لمانم ألوان اوستوالد وظلم لسكوبسن المركزي (Nassau, 2001).

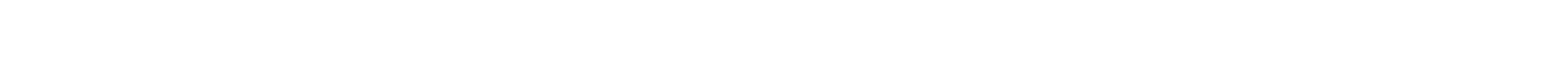

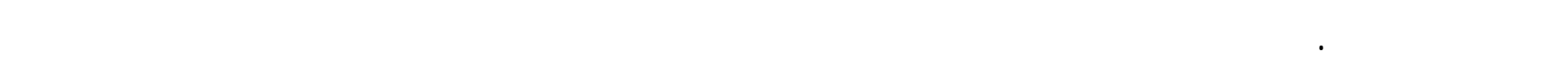

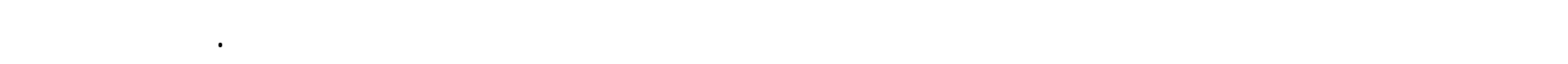

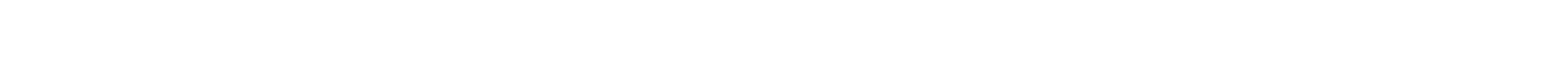
ومششحك توهين (Yakushenkov, 1980). إن مبشحكت الامتصاص لها أهمية كبيرة إذ بولنطتها يمكن لمتصاص الأطول الموجية للمنطق غير

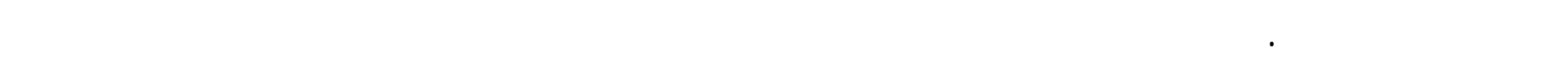

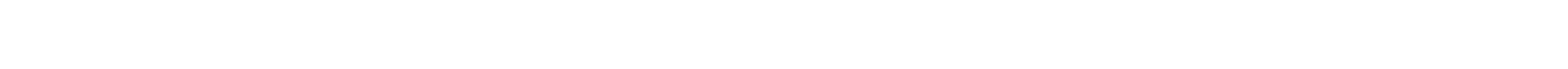

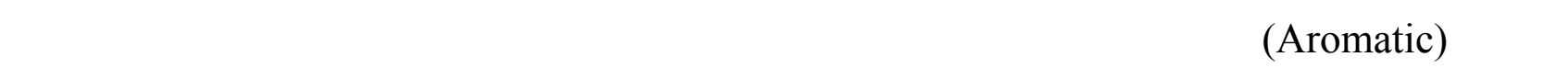
بلستخدل جيلات (Gels) صناعية ملونة. 


\section{Wratten Gelatin Filters}

مطشحت رلن جيلاتين

عبارة عن مبشحلت لمتصاصسهلة التصنبع رخيصة الثن وتصنع من إذلبة صبغلت عضوية معينة

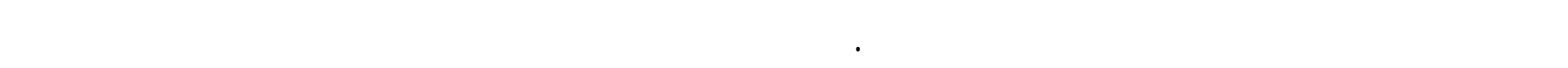
الضوئي والبصريك المجهرية.

الجزء القالري: (الضوئ والبصريك قولنين المتصاd

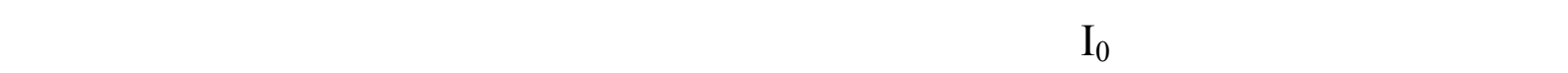

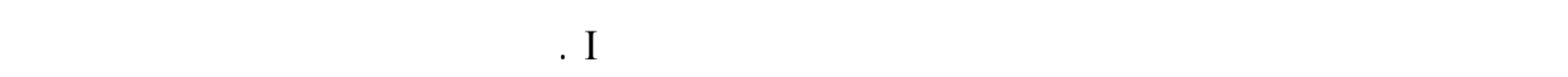

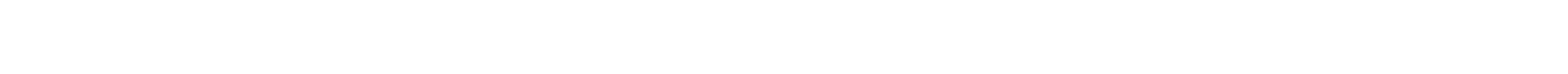

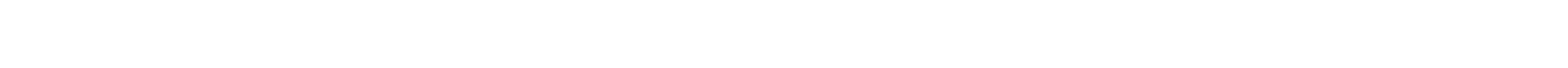
سيخضع لقانون لامبرت

$$
I=I_{0} e^{-\alpha \chi}
$$

حيث أن م معلمل الامتصاص وان xسمك المبشح

$$
\begin{gathered}
\log \frac{I}{I_{0}}=-\frac{\chi \alpha}{2.303} \\
\therefore A=\log \frac{I_{o}}{I}=\frac{\chi \alpha}{2.303}
\end{gathered}
$$

وبحذف اللثارة اللسالبة تصحح :

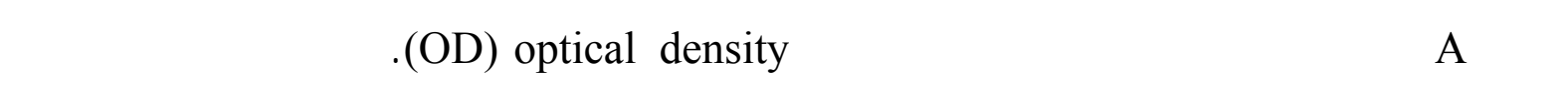

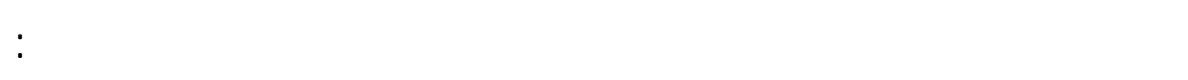

$$
A=\frac{C \chi \alpha}{2.303}
$$

تمل تركيز المحلول المحضر منه المششح. C

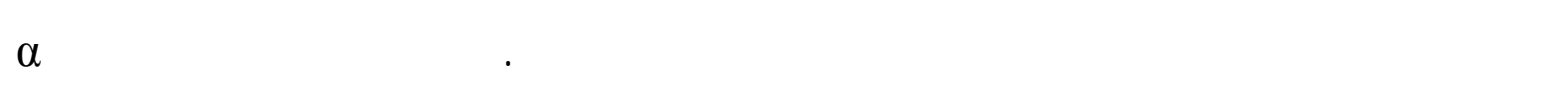
فتعتمد على الطول الموجي للضوء للستط وعلهطبية اللنيعة مرلكز الامتصاص. 


$$
\begin{aligned}
& \text { إن العلاقة بينشدة الضوء النفذ إلىشة الضوء للسفظ تسهى الفونية أي } \quad T=\frac{I}{I_{0}} \quad \text { أو: } \\
& A=-\log T=\log \frac{1}{T}
\end{aligned}
$$

\section{المتصاص الأشعة من فل الجزيئلت}

بما أن الأصباغ المستخلمة هي أصباغ عضوية وان ششوء اللون في هذه الأصباغ يفسر على لأس سلس

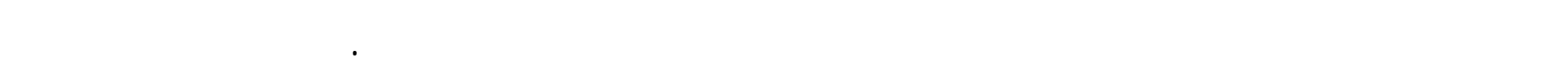

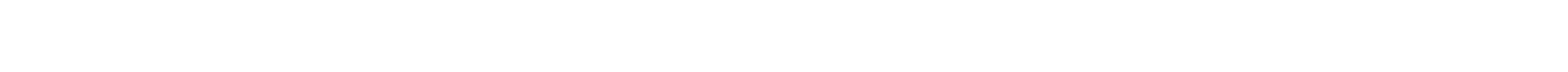

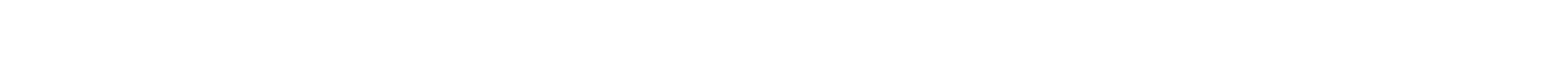

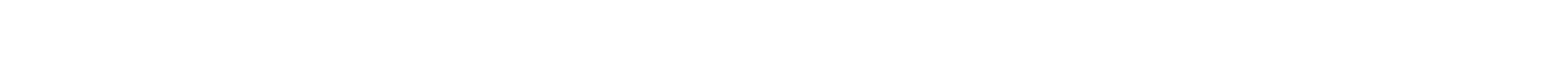
(1) يوضخ تغيرات الطلة للجزيئة.

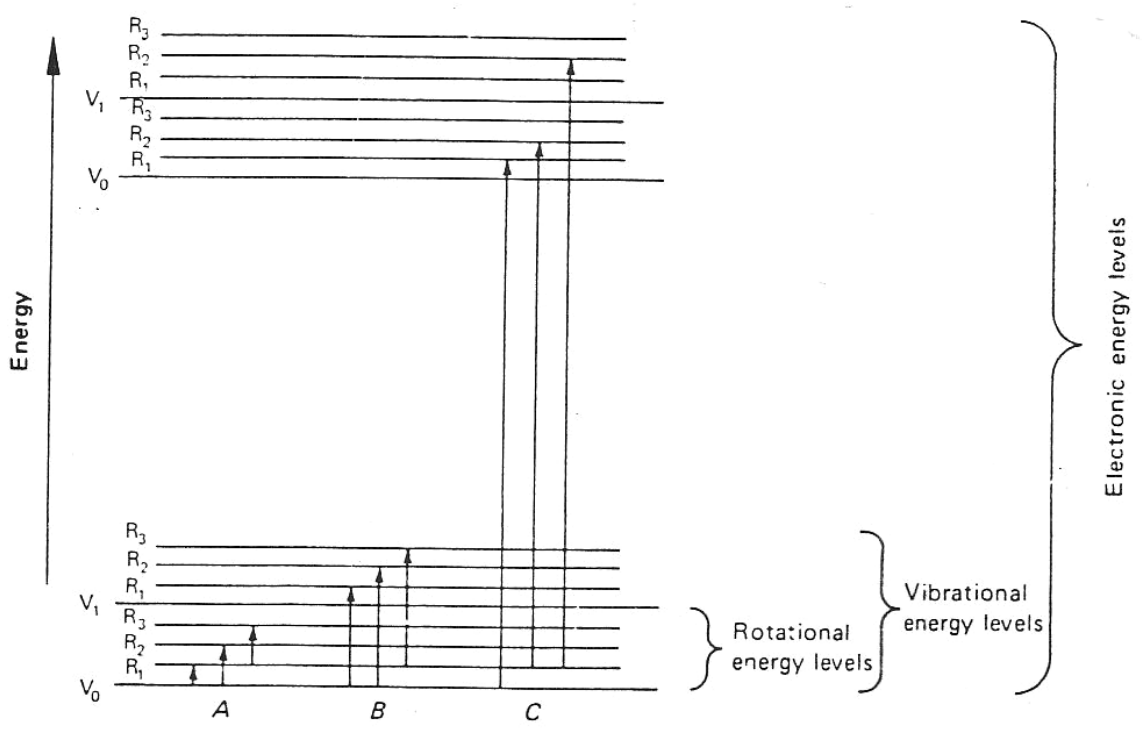

الشكل 1 : بسم تخطي الطاقة الجزيئية يوضح تغيرات الطاقة.

إن مستويت الطاقة الالكترونية لكبر من الاهتزازية والتي بدورها لكب ـرم ـن الدوراني ـة. تح حثث

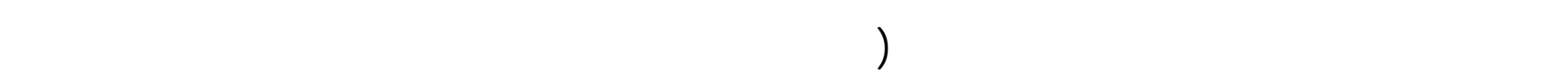

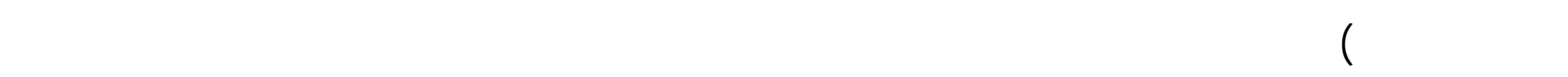
الانقالات الالكترونية فتحدث عندطاقة عالية أيضا في المطقة المرئية والفوق البفنجية (عبيد، 1988). 
الاتقالات الالكترونية تحدث نتيجة المتصاص الأشعة من قل مجلمبع ضمن الجزيئة تسمى الكروموف -ورات

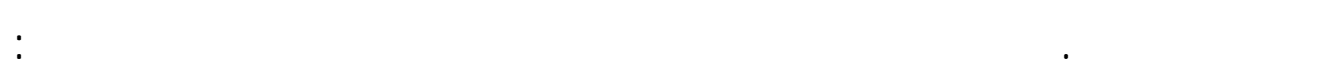

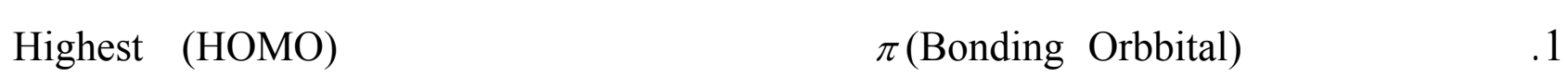
.Occupied Molecular Orbits 2. المدار المضاد للتآصر (Ant bonding orbital) أو يسم أولأ مدار جزيئي غير مشغول Lowest unoccupied Molecular orbital (LUMO).

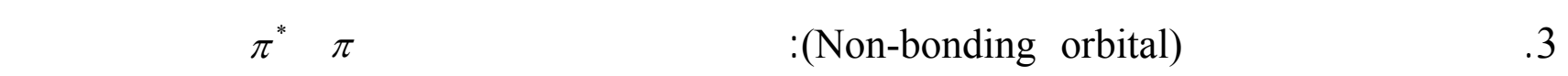
الكترونت لكنها لا تساهم بالتآصر في الجزيئة ويرمز له ( n ). إن أعلى المتصاص يحدث في الانق لـ لـ آل

$$
\pi \rightarrow \pi^{*}
$$

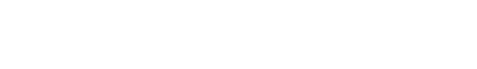

لغرض الحصول على هذه المعلملت التي توصف على المرشح يجب معرفة الاستجابة الطيفية والت ي

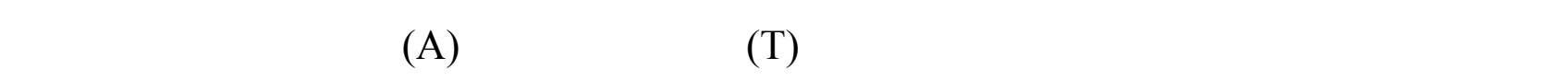

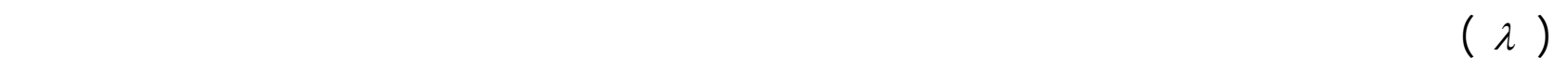
المعلملت التي توصف على المششح ، ومن هذه المعلمك:

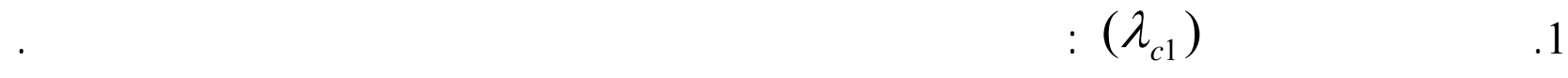

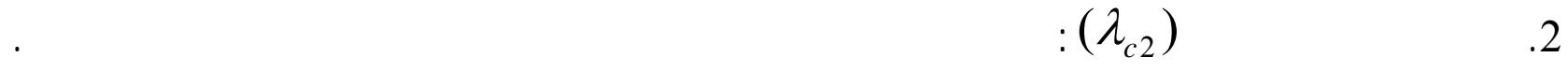
إن كلامن هنين المعلمين يعينان عند 5\% من الفانية الملقة (Spectrongon, comp, 2004). 3. مل ظلق فانية المششح: لمعرفة المنطق الحدوية التي قفطل الأطوال الموجية النافة على تلـ لـك المحجوبة بولنطة المرشح:

$$
\text { Slope } \%=\frac{\lambda\left(80 \% \text { of } T_{\max }\right)-\lambda_{c}}{\lambda_{C}} . \quad 100 \%
$$

4. عرض الطلق: يعرف على الهه العرض الكلل لطاق العبورمقلسا عندمتتصف الفاني ـة الق صصوى ويرaز له (FWHM). 5.

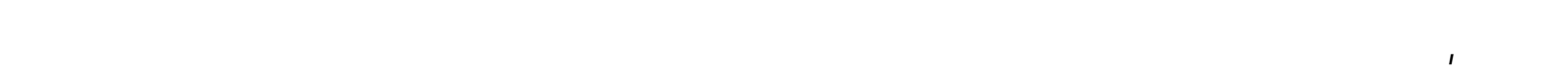
50\% من قمة الفانية.

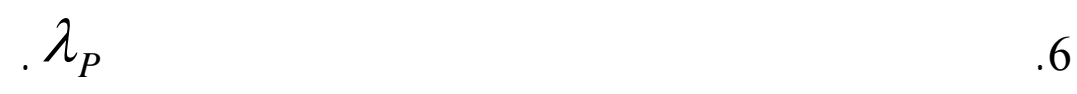




\section{الجزء الملي \\ تحضير النوذج:}

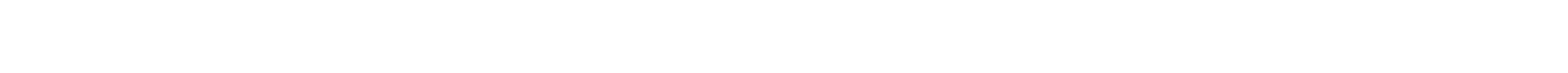

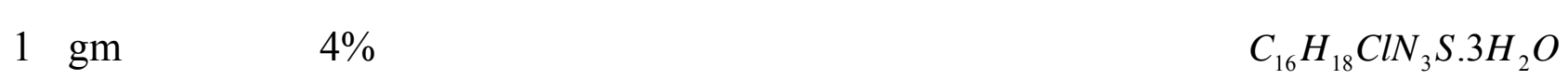

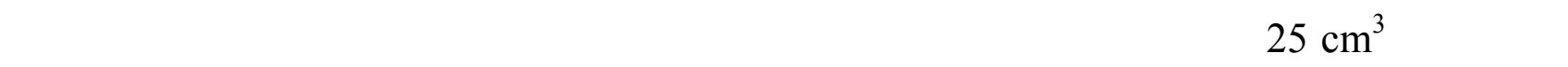
المحلول الجيلايتيني المى الوزن الطلوب من الصبغة بشكل تدريجي والمزج بهدوء ولمدة زمنية مختارة 20

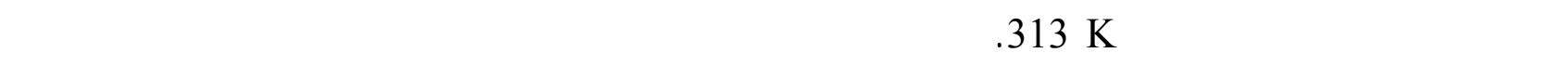

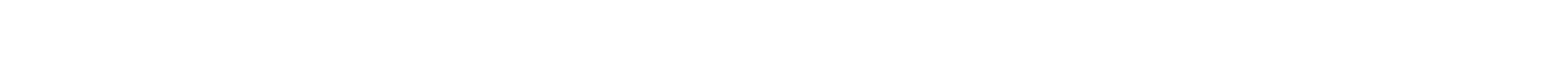

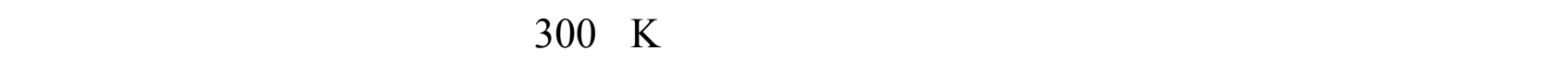

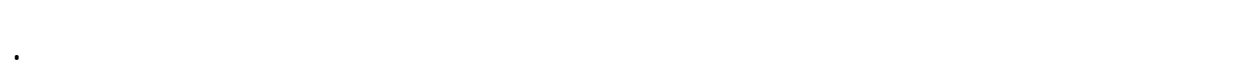

إلجراء الفيلسيك:

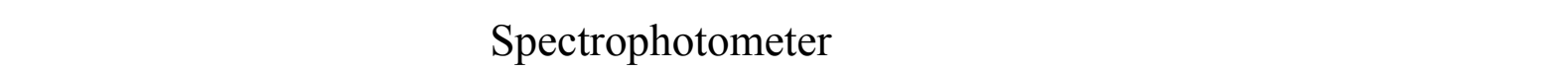

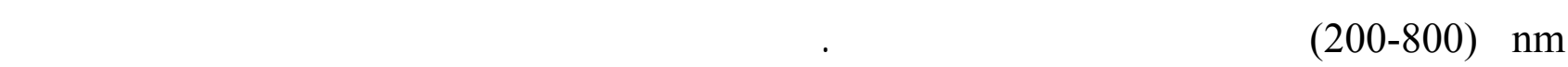
ومسلحة الغثاء.

\section{النتلئج والمنلثةة}

الشكل (2) يوضحطف الفانية لترلكيز مختلفة حيث لوظظ قصان الفانية بزياة التركي ـز وهـ ـذا

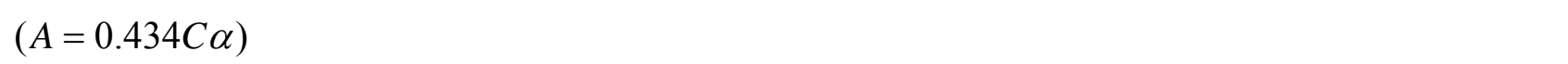
حيث C التركيز كما يلاظظ من الشكل حدوث حزم المتصاص قوية عند مطقة UV البعيدة وحزم المتصاص في مطقة UV القريبة. كما ولوحظ أيضا وجود حزم المتصاص عالية في المطقة المرئية للمدى (620-580)

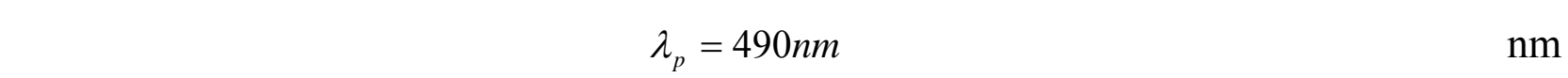

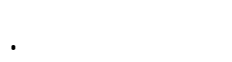




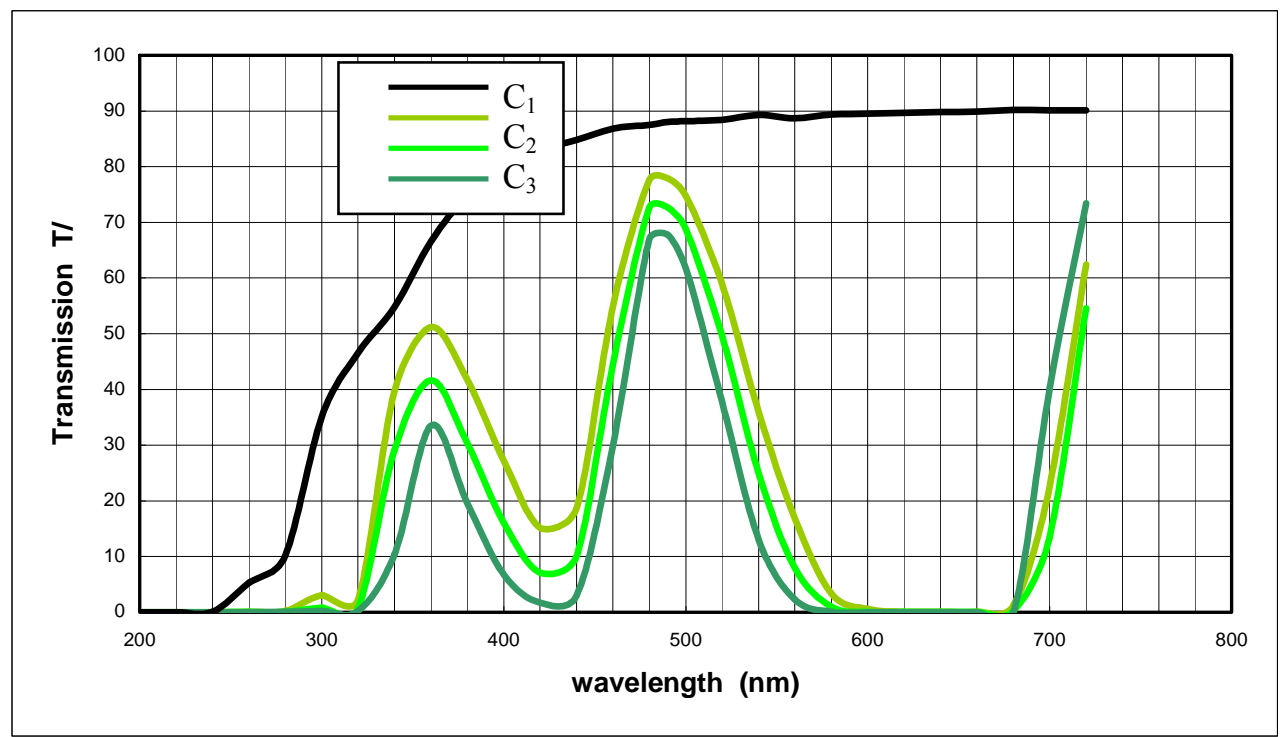

للشكل 2 : تغير منحني الاستجابة الطيفية الخاص بالفانية لمرشحلت راتن جيلاتين المصنعة من صبغة المثيل الأخضر بتغير تركي -ز الصبغ المة. $\mathrm{C}_{2}=0.28 \mathrm{gm} /$ liter $, \mathrm{C}_{3}=0.8 \mathrm{gm} / \mathrm{liter}, \mathrm{C}_{4}=1.2 \mathrm{gm} / \mathrm{liter}$ وبثبوتسمك المربح (x = $) .01 \mathrm{~cm}$.

الجدول (1) يبين الخواص البصرية لثلاثة مششحكت بترلكيز مختلفة مصنوعة م ـن صم ـبغة

المثل الأخضر.

الجدول 1 : الخواص البصرية لثلاثة مششحلت مختلفة في التركيزمصنوعة من صبغة المثل الأخضر.

\begin{tabular}{|c|c|c|c|}
\hline $\mathrm{x} / \mathrm{cm}$ & 0.01 & 0.01 & 0.01 \\
\hline $\mathrm{C} / \mathrm{gm} / \mathrm{Liter}$ & 0.28 & 0.8 & 1.2 \\
\hline $\mathrm{T}_{\max } \%$ & 77.9 & 72.7 & 67.8 \\
\hline$\lambda_{\mathrm{p}} / \mathrm{nm}$ & 490 & 490 & 490 \\
\hline $\mathrm{FWHM} / \mathrm{nm}$ & 85 & 75 & 6.5 \\
\hline $\mathrm{CWL} / \mathrm{nm}$ & 494.5 & 492.5 & 492 \\
\hline
\end{tabular}

للثكل (3) يوضح تغير المتصاصية المرشحك مع تركيز الصبغة بثب ـوت ال سمك عذ ـد الط ـول الم -وجي 施=490nm 


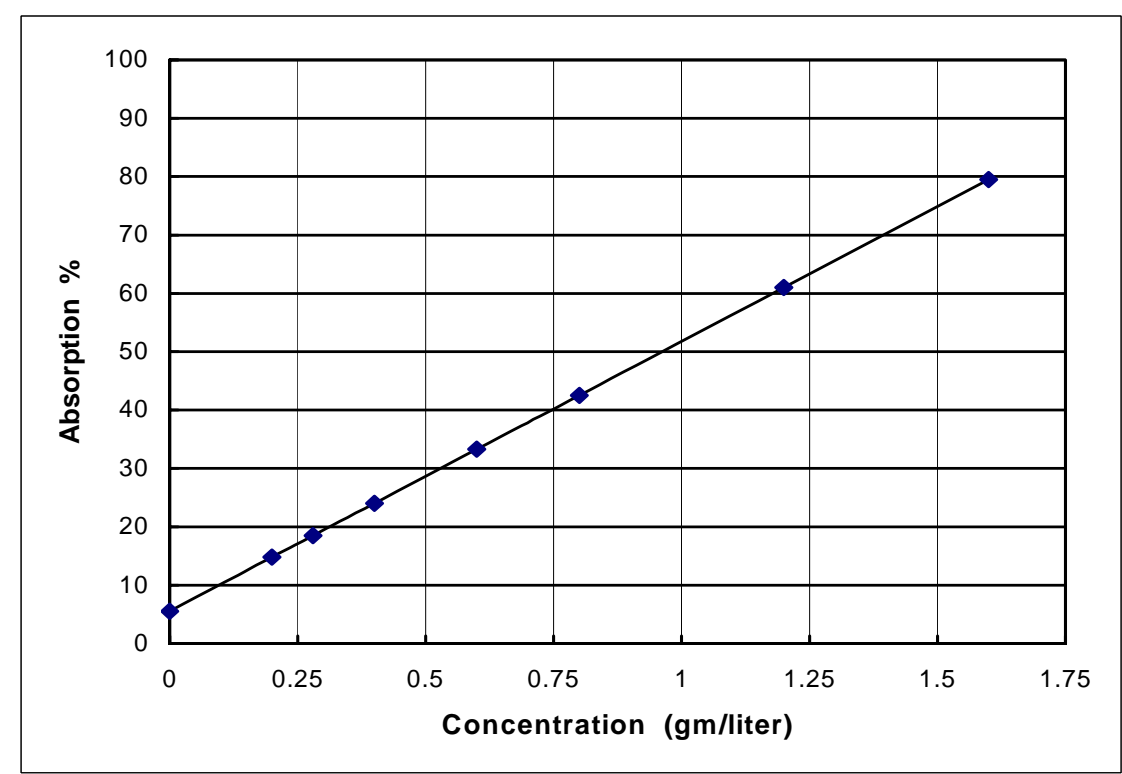

الشكل 3 : تغغير المتصاصية مششحك راتن جيلاتين مصنوعة من المثل الاخض -ر بتغير

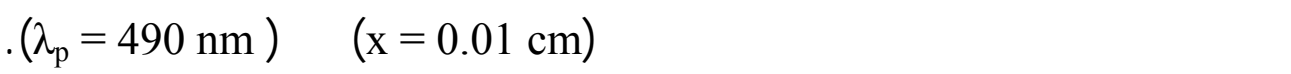

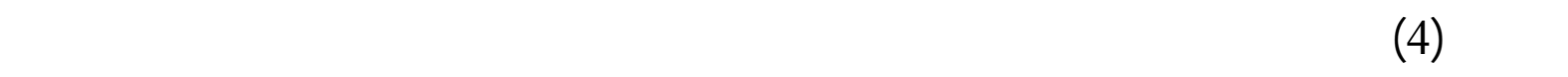

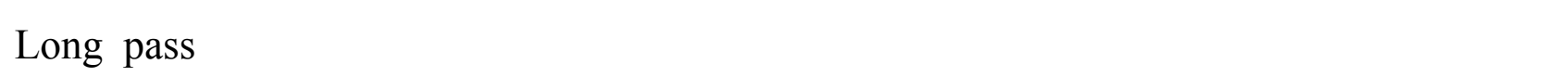

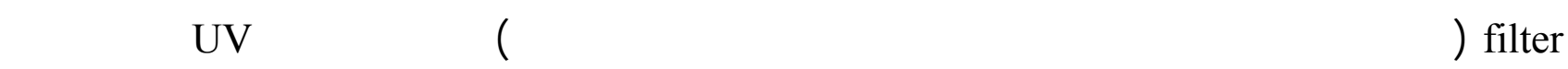

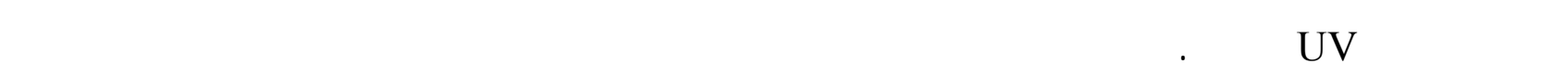
الزجاج لا يعد كروموجيناً.

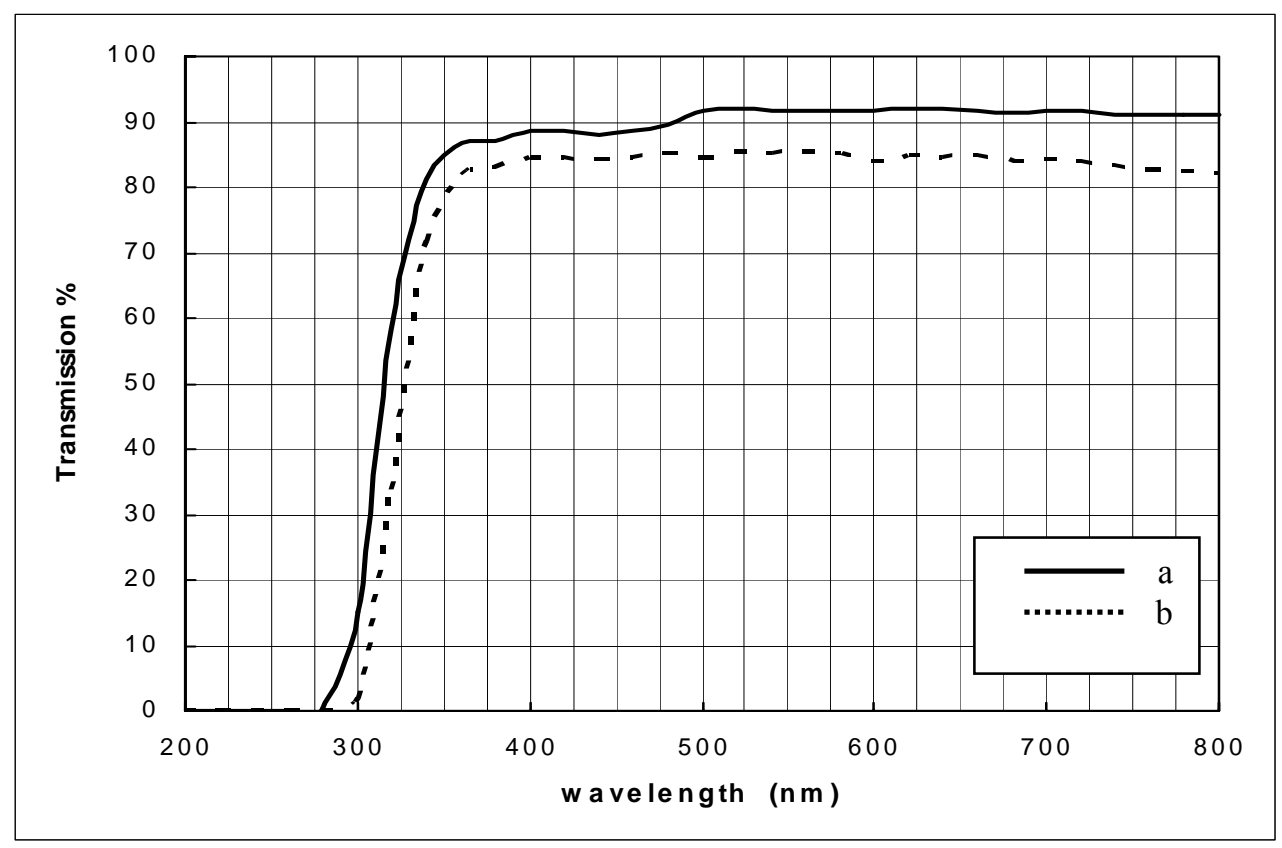

اللثكل 4 : منحني الإستجابة الطيفية a - لزجلجة ولحة b - لزجلجتين. 
الثشل (5) يوضح تأثير زجلجتي الحماية على منحف الاستجابة الطفية للمششح إذ يظهر أن زجلجة الحماية

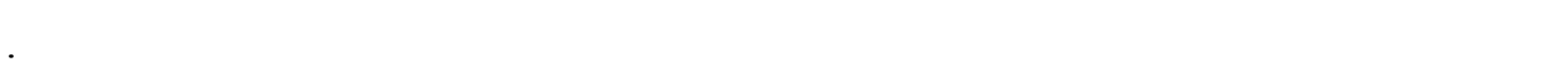
اللشكل (6) يمل زمن تأثير زمن التعتق على الخواص البصرية ولفترة زمنية مقدارها أربعة ألشهر وقد تبين عدم وجود تأثير على الخواص البصرية من خلل عملية الخزن.

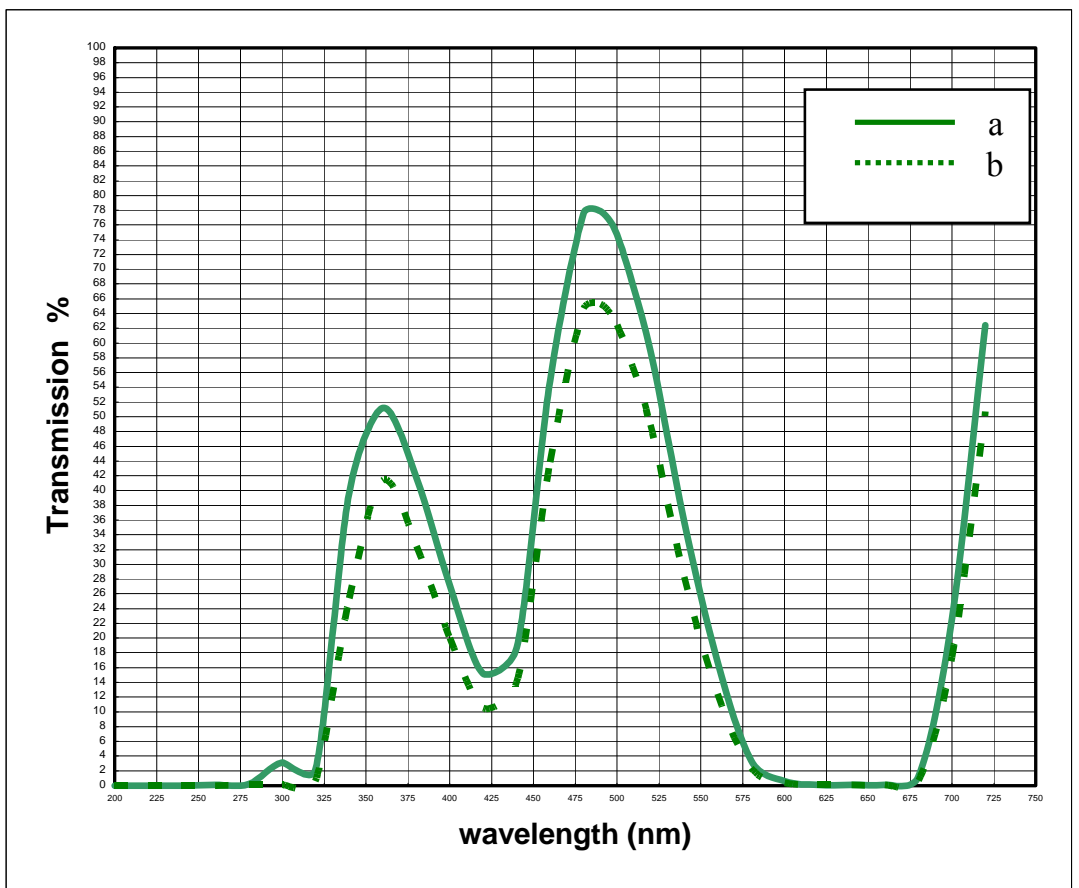

اللشكل 5: تأثير زجلجتي الحماية على منحني الإتجابة الطفية لمرشح رالتن جيلاتين

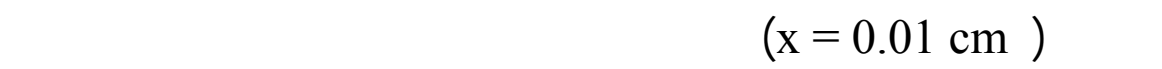

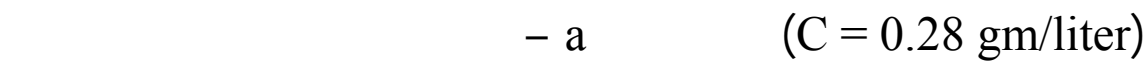

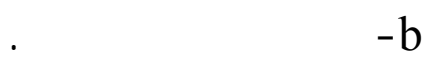




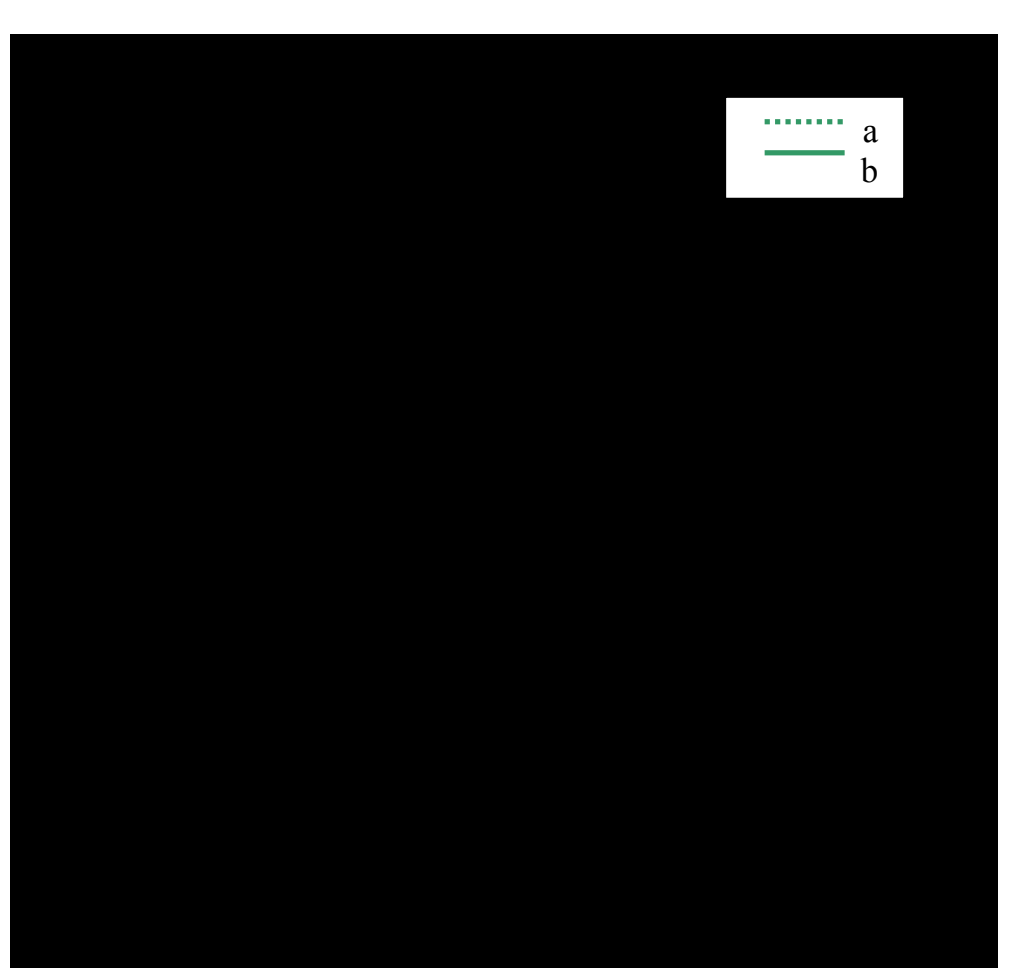

الشكل 6 : تأثير زمن التعتيق على الخواص البصرية لمبش ـحمصنوع من صبغ ـة

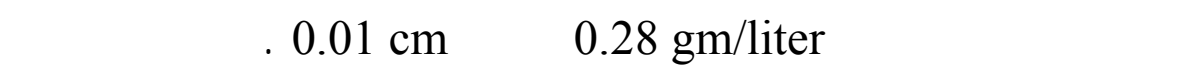
a - لخذت بعد التصنيع بيومن b - الخذ بعد مرور أربعة أشهر من التصنيع.

وأخيرا للشكل (7) يوضح الظاقق الكبير لمنحن الاستجابة الطيفية للمششح المصنع والمششح القيلسي رقم (44) المصنع من قبلشركة لستملن كوداك والمنكور في المصدر (Weast and Astle,1979)

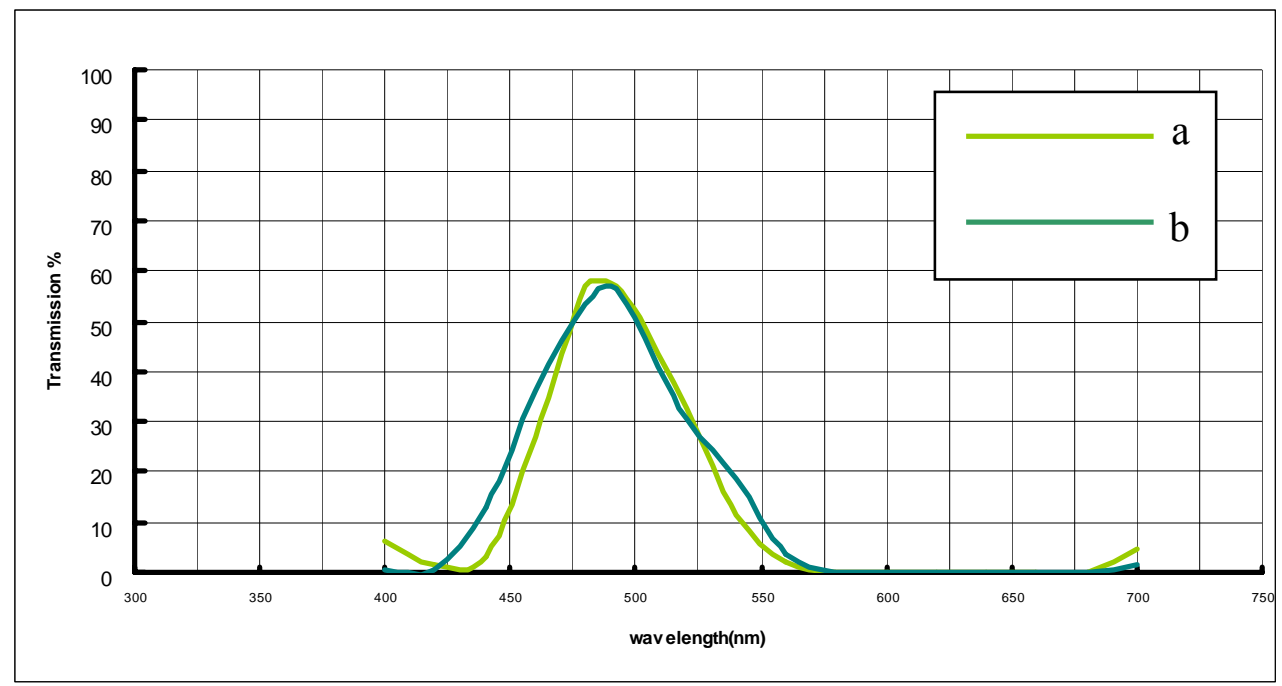

اللشكل 7 : منحنيا لمتجابة . a - لمرشح مصنع من صبغة المثل الأخضر بتركيز صبغة (C) = 1.20 gm/liter)

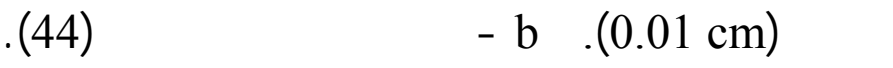




\section{الاستنتاجلت}

من خلال الدرلسة للخواص البصرية للمرشح المصنع يمكن لستنتاج ما يلي:

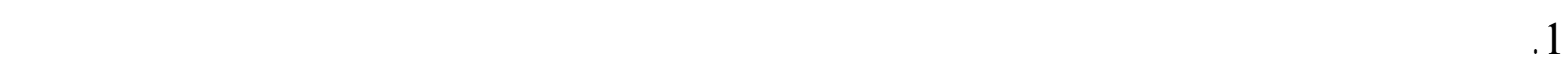
الضوئي. والبصريك المجهرية 2. ت تغير الخواص البصرية للمرشح بتغير تركيز صبغته.

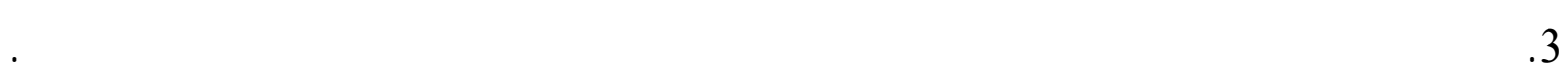

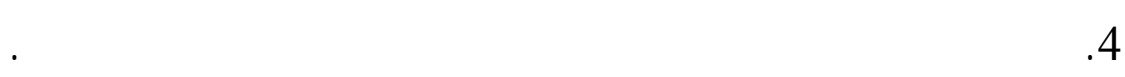

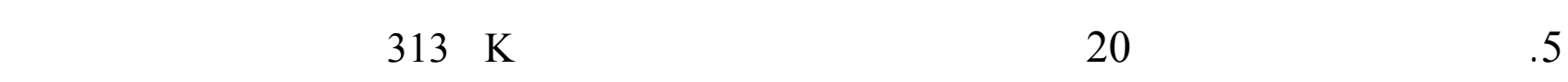
تجفيف فكانت

\section{المصاكر المربية}

عبيد، فتحي لحميد، (1988). "طرق التحليل الآلي". جلمعة الموصل، دار الكتب للطباعة والذ شر، جلمع ـة الموصل.

\section{المصاكر لأجنبية}

Nassau, K. (2001)." The Physics and Chemistry of Color" . Published by John Wiley and Sons, Inc.

Serway, J. (2003)." Physics for Scientists and Engineers with Modern Physics". 6th edn. Thomson.

Spectrongon, Comp. (2004). "Optical Interference Filters", 17th edn., WWW. Spectrongon. Comp. p.15.

Tilley, R. (2000)." Color and the Optical Properties of Materials". John Wiley and Sons, Ltd.

Weast, R. C. ; Astle, M. J. (1979)." CRC Handbook of Chemistry and Physics". 60 edn., CRC, press.

Yakushenkav, Yu. (1980)."Electro-optical Devices". Mir publishers, Moscow. 